\title{
Development of a canine model with diffuse hepatic vein obstruction (Budd-Chiari syndrome) via endovascular occlusion
}

\author{
BIN SHEN, QINGQIAO ZHANG, XIAOLONG WANG, HAO XU, MAOHENG ZU, \\ MENG WU, ZHIKANG GAO, WENLIANG WANG, JINCHANG XIAO and YONG WANG \\ Department of Interventional Radiology, The Affiliated Hospital of Xuzhou Medical College, \\ Xuzhou, Jiangsu 221006, P.R. China
}

Received June 15, 2013; Accepted November 22, 2013

DOI: $10.3892 / \mathrm{mmr} .2013 .1868$

\begin{abstract}
The aim of the present study was to develop a reliable and reproducible canine model to mimic human diffuse hepatic vein obstruction (Budd-Chiari syndrome, BCS). A total of 24 canines were divided into an experimental $(n=18)$ and a control $(n=6)$ group. Under the guidance of digital subtraction angiography, a balloon catheter was delivered to the target hepatic vein (the common trunk of the left hepatic and middle hepatic veins) via the right external jugular vein. The balloon was inflated to completely block the vessels. For the canines in the experimental group, a mixture of $N$-butyl-cyanoacrylate (NBCA) and lipiodol (3-5 ml) was injected via the balloon catheter. Canines in the control group were injected with equal volumes of normal saline. Liver function and pathology were examined at 4, 6 and 8 weeks following surgery. BCS was successfully established in all members of the experimental group and there were no serious complications in either group. The left and middle hepatic veins and common trunk were completely obstructed at 4, 6 and 8 weeks following surgery in the experimental group, while in the control group, the hepatic vein remained unobstructed at 4 weeks. There was hepatocyte congestion and edema at 4 weeks following surgery in the experimental group and the edema became aggravated following 6 weeks. At 8 weeks following surgery, there was necrosis of hepatocytes and significant thickening of the hepatic vein tunica intima in addition to an increased number of elastic fibers. In conclusion, the present study demonstrates that a reliable and reproducible canine model of BCS can be developed by endovascular obstruction of the hepatic vein.
\end{abstract}

Correspondence to: Dr Qingqiao Zhang, Department of Interventional Radiology, The Affiliated Hospital of Xuzhou Medical College, 99 Huaihai West Road, Xuzhou, Jiangsu 221006, P.R. China

Email: 1427286069@qq.com

Key words: animal model, Budd-Chiari syndrome, hepatic vein, portal hypertension, hepatic function

\section{Introduction}

Budd-Chiari syndrome (BCS) is a life-threatening disorder characterized by hepatic venous outflow obstruction at any level from the small hepatic veins to the cavoatrial junction $(1,2)$. According to possible sites of obstruction, BCS may be divided into hepatic vein obstruction, inferior vena cava obstruction and a mixture of hepatic vein and inferior vena cava obstructions. Development of effective vascular surgery and interventional treatment approaches for the treatment of BCS associated with diffuse hepatic vein obstruction is difficult $(3,4)$. Multiple studies have shown that portal vein-vena cava surgical shunt does not increase the survival of BCS patients (5-8). Normal hepatic vein flow cannot be recovered in patients with BCS associated with chronic diffuse hepatic vein obstruction, therefore, balloon dilation and stenting is not suitable for this type of BCS. Transjugular intrahepatic portosystemic shunts (TIPS) are able to relieve liver congestion and improve clinical symptoms but the occurrence of TIPS dysfunction and hepatic encephalopathy remains high (9). In order to more effectively treat these symptoms, it is necessary to establish an animal model of BCS to facilitate the development of surgical or interventional approaches. A mouse model of BCS was previously established by ligation of the inferior vena cava at the proximal end $(10,11)$. However, this model used a surgical approach in which hepatic vein flow disorder was caused by stenosis or occlusion of the inferior vena cava near the right atrium, rather than by diffuse occlusion of the hepatic vein. At present, there is no animal model in place mimicking human BCS associated with diffuse hepatic vein occlusion. The present study used an endovascular technique to establish a reliable and reproducible canine model of this BCS type. This model may be useful for future pathophysiological, surgical and interventional treatment studies of BCS.

\section{Materials and methods}

Grouping of the animals. A total of 24 healthy adult mongrel canines between 1 and 3 years-old $(1.95 \pm 0.65$ years-old; male and female; weight, $18 \pm 2.3 \mathrm{~kg}$; Experimental Animal Center of Xuzhou Medical College, Xuzhou, China) were randomly divided into experimental $(n=18)$ and control $(n=6)$ groups. The canines were preconditioned in the laboratory (Experimental 
Animal Center of Xuzhou Medical College) for several weeks and received standard dog chow containing $25 \%$ protein and $8 \%$ fat. The same diet was used postoperatively. Animal handling complied with the regulations on the management of laboratory animals of the Chinese Academy of Sciences and the study was approved by the Ethics Committee of Xuzhou Medical College, Xuzhou, China.

Procedure for model development. All animals were fasted $8 \mathrm{~h}$ prior to surgery and skin preparation was performed on the right side of the neck. General anesthesia was performed by intramuscular injection of 3\% sodium pentobarbital $(1 \mathrm{ml} / \mathrm{kg}$; Hongyun Long Biological Technology Co. Ltd., Wuhan, China) without endotracheal intubation. Canines were fixed in the supine position on the digital subtraction angiography (DSA) examination bed (Innova 3100; GE Healthcare, Little Chalfont, UK). The right side of the neck was disinfected with iodophor. Each layer of skin was cut along the outer edge of the right sternocleidomastoid to expose the right external jugular vein. Puncture of the right external jugular vein was performed by modified Seldinger technique (12). A 6F-catheter sheath was delivered along a short guidewire and, under the guidance of DSA, a $5 \mathrm{~F}$ angiographic catheter $\left(\mathrm{TEMPO}^{\circledR} 4\right.$; Cordis Corporation, East Bridgewater, NJ, USA) was delivered to the left, middle and right hepatic veins for phlebography. A contrast agent (iohexol) was injected at a flow rate of $3.0 \mathrm{ml} / \mathrm{sec}$ and a total volume of $6.0 \mathrm{ml}$. Images were captured at 4 frames/sec to show the hepatic vein diameter and degree of reflux. The hepatic vein with maximum diameter was selected as a target for placement of the exchange guidewire. The balloon catheter (diameter, 8-12 mm; Cordis Corporation) was delivered to the target hepatic vein along the guidewire and the balloon was filled with contrast agent. Once complete occlusion of blood flow in the hepatic vein had been confirmed, an emulsion mixture (3-5 ml) of $N$-butyl-cyanoacrylate (NBCA; Baiyun Medical Adhesive Co. Ltd., Guangzhou, China) and lipiodol (2:1; Guerbet, Villepinte, France) was injected into animals of the experimental group via the balloon catheter. Injections were stopped upon observation of vascular casting of the hepatic vein by X-ray. Subsequently, the balloon catheter was removed and a 5F-angiographic catheter was placed to indicate hepatic vein occlusion. In the control group, $3-5 \mathrm{ml}$ normal saline was injected via balloon catheter and contrast agent was injected to indicate hepatic vein patency. Following surgery, the catheter and sheath were removed and the external jugular vein pressed to ensure that no bleeding occurred. Subsequently, each layer of the incision was sutured and disinfected. Antibiotics (penicillin, $800,000 \mathrm{U} /$ day) and analgesics (ketoprofen, $1 \mathrm{ml} / 5 \mathrm{~kg}$ ) were used for a period of three days postoperatively. The animals were then returned to their breeding circle and their airways were kept open. Breathing and eating patterns were monitored daily. Following surgery, the animals were allowed unlimited exercise.

Sample collection and biochemical testing. Under general anesthesia, peripheral venous blood was collected from six canines in the control group (at 4 weeks following surgery) and 18 canines from the experimental group (six from each of the 4-, 6- and 8-week postoperative stages). The blood samples were kept in procoagulant test tubes and centrifuged at $4^{\circ} \mathrm{C}$ for $10 \mathrm{~min}(1,600 \mathrm{x} \mathrm{g}$ or $3,000 \mathrm{rpm})$. The serum was tested for alanine aminotransferase, $\gamma$-glutamyl transpetidase, albumin, pro-albumin, total bile acid, total bilirubin and cholinesterase.

Color Doppler ultrasound evaluation. Portal venous hemodynamics, proper hepatic arterial hemodynamics and sonographic appearance of liver parenchyma were evaluated with color Doppler ultrasound and two-dimensional gray-scale ultrasound using a curvilinear $3.5 \mathrm{MHz}$ transducer (iU22; Philips, Eindhoven, Netherlands). Canines were examined at supine and lateral positions under general anesthesia. Diameter of main portal vein (D), average peak velocity (V), diameter of proper hepatic artery, peak systolic velocity (PSV) and end-diastolic peak velocity (EDV) were measured over a period of 4-5 cardiac cycles. Average values of these parameters were obtained from continuous measurements taken three times. Resistance index (RI) and blood flow volume (Q) were calculated according to the following formulae: $\mathrm{RI}=(\mathrm{PSV}-\mathrm{EDV}) / \mathrm{PSV} ; \mathrm{Q}=\mathrm{V} \times(\mathrm{D} / 2)^{2} \times \pi \times 60$, respectively.

Measurement of portal venous pressure. The abdominal cavity was cut along the right quarter of the costal arch. Portal venous pressure (PVP) was determined by cannulation of the superior mesenteric vein. The unit of measurement was $\mathrm{cm} \mathrm{H}_{2} \mathrm{O}$ $\left(1 \mathrm{~cm} \mathrm{H}_{2} \mathrm{O}=0.098 \mathrm{kPa}\right)$.

Harvest of liver specimen. Gross anatomical changes were recorded following the sacrifice of the animals by laparotomy. The target hepatic vein and hepatic tissues from the drainage area of the target hepatic vein were collected and fixed with $10 \%$ formalin. Hematoxylin and eosin (H\&E) staining was performed to analyze structural changes in hepatic cells and the hepatic vein wall using an SZX16 microscope (Olympus, Tokyo, Japan). The results were compared between experimental and control groups.

Statistical analysis. The SPSS 16.0 package (SPSS, Inc., Chicago, IL, USA) was used for statistical analysis. Data measurements are expressed as mean \pm standard deviation. One-way analysis of variance was used to compare the data between different groups and $\mathrm{P}<0.05$ was considered to indicate a statistically significant difference.

\section{Results}

Angiographic performance during surgery. Hepatic venography indicated that the common trunk of left hepatic and middle hepatic veins had the largest diameter. This was therefore used as a target hepatic vein. Obstruction occurred in the target hepatic vein of all 18 canines in the experimental group (Figs. 1 and 2), indicating a 100\% success rate of model development. The hepatic vein in the control group was unobstructed. There were no serious complications (e.g., pulmonary embolism or mortality) in either group.

Liver function in the experimental and control groups is shown in Table I.

Clinical symptoms and signs of animals in the experimental group. In the experimental group, anorexia and hepatomegaly occurred in 18 canines following surgery. Ascites occurred in 3, 5 and 5 canines at 4,6 and 8 weeks following surgery, 
Table I. Liver function of canines in experimental and control groups (mean $\pm \mathrm{SD} ; \mathrm{n}=6$ ).

\begin{tabular}{|c|c|c|c|c|}
\hline \multirow[b]{2}{*}{ Parameter } & \multicolumn{3}{|c|}{ Experimental group } & \multirow{2}{*}{$\begin{array}{l}\text { Control } \\
\text { group }\end{array}$} \\
\hline & 4 weeks & 6 weeks & 8 weeks & \\
\hline ALT, U/1 & $52.50 \pm 12.50^{\mathrm{a}}$ & $61.30 \pm 5.70^{\mathrm{a}}$ & $38.60 \pm 9.40^{\mathrm{a}}$ & $28.60 \pm 5.30$ \\
\hline GGT, U/1 & $3.60 \pm 2.40$ & $4.00 \pm 2.00$ & $3.80 \pm 1.30$ & $3.50 \pm 2.70$ \\
\hline $\mathrm{PAB}, \mathrm{g} / 1$ & $0.18 \pm 0.04^{\mathrm{a}}$ & $0.22 \pm 0.02^{\mathrm{a}}$ & $0.19 \pm 0.06^{\mathrm{a}}$ & $0.26 \pm 0.13$ \\
\hline ALB,$g / 1$ & $35.20 \pm 6.80$ & $29.40 \pm 2.30^{\mathrm{a}}$ & $34.70 \pm 8.70$ & $34.50 \pm 3.50$ \\
\hline $\mathrm{TBIL}, \mu \mathrm{mol} / \mathrm{l}$ & $0.90 \pm 0.50$ & $1.20 \pm 0.20$ & $1.40 \pm 0.50$ & $1.10 \pm 0.30$ \\
\hline $\mathrm{TBA}, \mu \mathrm{mol} / \mathrm{l}$ & $0.85 \pm 0.35$ & $0.90 \pm 0.25$ & $1.15 \pm 0.45$ & $0.93 \pm 0.47$ \\
\hline CHE, IU/l & $4,595.30 \pm 59.60^{\mathrm{a}}$ & $5,135.50 \pm 84.50^{\mathrm{a}}$ & $4,983.50 \pm 123.50^{\mathrm{a}}$ & $5,327.60 \pm 75.30$ \\
\hline
\end{tabular}

${ }^{\mathrm{a}} \mathrm{P}<0.05$ vs. the control group. ALT, alanine aminotransferase; GGT, $\gamma$-glutamyl transpeptidase; PAB, pro-albumin; ALB, albumin; TBIL, total bilirubin; TBA, total bile acid; CHE, cholinesterase.

Table II. Hepatic hemodynamics of canines in experimental and control groups (mean \pm SD; $n=6$ ).

\begin{tabular}{|c|c|c|c|c|}
\hline \multirow[b]{2}{*}{ Parameter } & \multicolumn{3}{|c|}{ Experimental group } & \multirow{2}{*}{$\begin{array}{c}\text { Control } \\
\text { group }\end{array}$} \\
\hline & 4 weeks & 6 weeks & 8 weeks & \\
\hline Diameter of PV, cm & $0.97 \pm 0.05$ & $0.99 \pm 0.13$ & $0.91 \pm 0.17$ & $1.10 \pm 0.08$ \\
\hline Average peak velocity of $\mathrm{PV}, \mathrm{cm} / \mathrm{sec}$ & $27.10 \pm 2.10^{\mathrm{a}}$ & $19.70 \pm 2.50^{\mathrm{a}}$ & $20.20 \pm 1.50^{\mathrm{a}}$ & $29.70 \pm 3.30$ \\
\hline $\mathrm{BFV}$ of $\mathrm{PV}, \mathrm{ml} / \mathrm{min}$ & $1,301.60 \pm 73.60^{\mathrm{a}}$ & $989.40 \pm 42.20^{\mathrm{a}}$ & $1,187.90 \pm 35.20^{\mathrm{a}}$ & $1,561.70 \pm 64.20$ \\
\hline $\mathrm{PVP}, \mathrm{cm} \mathrm{H}_{2} \mathrm{O}$ & $16.50 \pm 2.50^{\mathrm{a}}$ & $17.30 \pm 1.20^{\mathrm{a}}$ & $16.70 \pm 2.30^{\mathrm{a}}$ & $11.30 \pm 1.60$ \\
\hline Diameter of PHA, cm & $0.30 \pm 0.02$ & $0.28 \pm 0.03$ & $0.31 \pm 0.02$ & $0.31 \pm 0.03$ \\
\hline RI of PHA & $0.68 \pm 0.03^{\mathrm{a}}$ & $0.68 \pm 0.04^{\mathrm{a}}$ & $0.66 \pm 0.21^{\mathrm{a}}$ & $0.57 \pm 0.16$ \\
\hline
\end{tabular}

${ }^{\mathrm{a}} \mathrm{P}<0.05$ vs. control group. PV, portal vein; BFV, blood flow volume; PVP, portal venous pressure; PHA, proper hepatic artery; RI, resistance index.

respectively. At 6 weeks post-surgery, one canine exhibited upper gastrointestinal bleeding.

Color Doppler ultrasound observations. In the experimental group, no color Doppler signal was observed in the left and middle hepatic veins or in their common trunk. Reverse flow signaling occurred in the distal segment of the left and middle hepatic veins at 4, 6 and 8 weeks following surgery. A heterogeneous hypoechoic mass was observed in the occluded area of the liver. The color Doppler signal in the main portal vein changed from hepatopetal to hepatofugal flow in two canine BCS models. In 16 canine BCS models, the signal remained hepatopetal but weakened markedly. In the control group, the patent hepatic veins were confirmed by color Doppler ultrasound and the results showed that the flow maintained normal hepatopetal character in the main portal vein and the liver showed homogeneous isoecho at week 4 following surgery. Altered hemodynamics of the portal vein and proper hepatic artery are shown in Table II.

Gross anatomy in experimental and control groups. In the experimental group, the left and middle hepatic veins and the common trunk were filled with solidified embolic agents at 4 ,

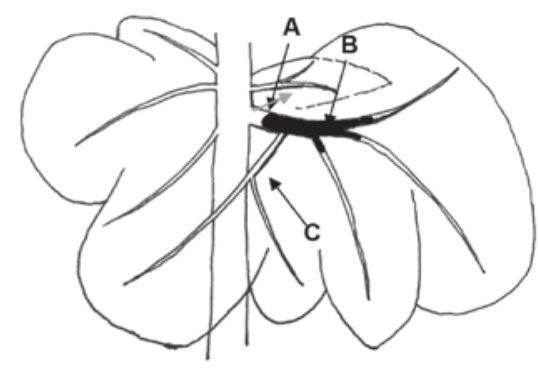

Figure 1. Sketch showing hepatic venous anatomy of canine Budd-Chiari syndrome model and the site of occlusion (arrows). (A) Common trunk of left hepatic and middle hepatic veins, (B) left hepatic vein and (C) middle hepatic vein.

6 and 8 weeks following surgery and the lumen was completely obstructed. The liver exhibited swelling at the draining area and a color of pale red, dark red and near black was noted at 4, 6 and 8 weeks following surgery, respectively (Fig. 3A). In the control group, the hepatic vein was patent and the liver was bright red with a smooth surface and sharp edge (Fig. 3B).

Changes in liver pathology in the experimental and control groups. Four weeks after surgery, animals in the control 


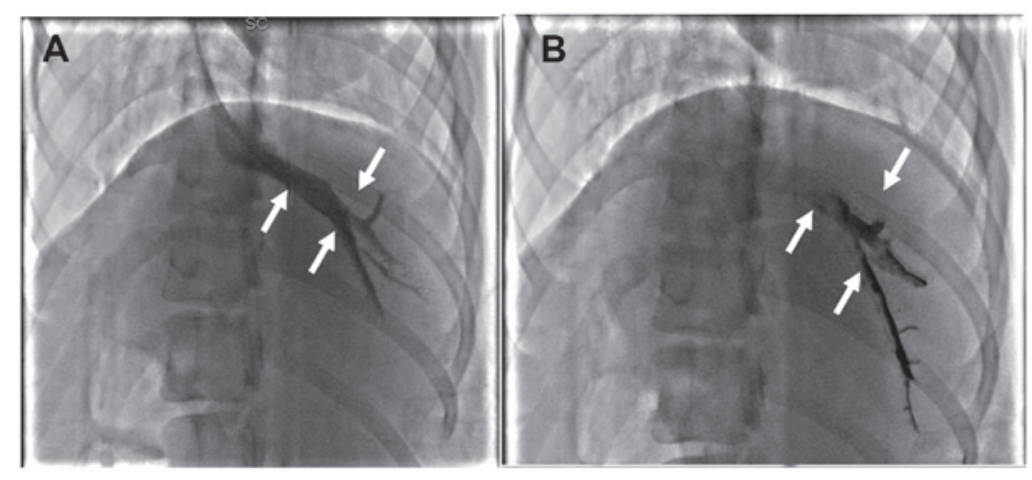

Figure 2. Venography observations of canine Budd-Chiari syndrome model. (A) Preoperative venography-demonstrated patency of left and middle hepatic veins and common trunk (arrows). (B) Postoperative fluoroscopy of digital subtraction angiography-detected vascular casting of left and middle hepatic veins and common trunk (arrows).

group exhibited normal hepatic cell structure and hepatic veins (Fig. $4 \mathrm{~A}_{1}$ and $\mathrm{A}_{2}$ ). However, light microscopy revealed hepatic cell congestion, edema, dilated centrilobular sinusoids, widened central veins in the hepatic lobules, red blood cells situated in Disse's space and a large quantity of inflammatory cell infiltration (in the tunica intima and tunica media of the hepatic vein without significant thickening) in the experimental group at week 4 following surgery (Fig. $4 \mathrm{~B}_{1}$ and $\mathrm{B}_{2}$ ). At week 6, hepatic cells showed swelling with lipid degeneration and a number of neutrophils, Kupffer cells and macrophages (Fig. $4 \mathrm{C}_{1}$ ). The hepatic vein tunica intima showed marginal thickening and the circular smooth muscle of the tunica media had become thickened with an increased number of layers (Fig. $4 \mathrm{C}_{2}$ ). At week 8 following surgery, the majority of hepatic cells exhibited balloon-like changes with sporadic atrophy of multiple hepatic cells, necrosis, lightly stained cytoplasm, enlarged nucleolus, increased number of polyploid nuclei cells and reduced inflammatory infiltration compared with that at week 6 (Fig. $\left.4 \mathrm{D}_{1}\right)$. The hepatic vein intima exhibited significant thickening with hyperplasia of the tunica media and elastic fibers (Fig. $4 \mathrm{D}_{2}$ ).

\section{Discussion}

The present study successfully established a canine BCS model by obstruction of the common trunk of the left and middle hepatic veins and intravenous injection of an NBCA-lipiodol mixture. Hepatomegaly, varying degrees of hepatic dysfunction and altered hemodynamics of the portal vein (e.g., weakened hepatopetal color Doppler signal, decreased portal blood volume and increased PVP) were observed in 18 canine BCS models at 4-8 weeks following surgery. Ascites was observed in 13 of 18 canine models. Therefore, all canine models in the present study exhibited the classic symptoms and pathology of BCS.

Balloon obstruction of hepatic veins in humans has been successfully achieved through the right femoral vein, right and left femoral veins, the right internal jugular vein and percutaneous liver pathways (13). In contrast to humans, the external jugular vein in canines has a greater diameter $(4.5-5.0 \mathrm{~mm})$ than the internal jugular vein. Furthermore, this vein is more superficially located, being covered only by nuchal muscle. The carotid vein has a larger diameter but is located in a deeper position, which is not conducive to surgical intervention. Therefore, the external jugular vein was selected and a $100 \%$ rate of technical success was achieved. An NBCA-lipiodol mixture has previously been used as the embolic agent for the treatment of symptomatic polycystic liver disease, intramuscular active hemorrhage and tumors (14-16). However, use of the NBCA-lipiodol mixture for the development of BCS animal models has not yet been reported. Attention to the speed and time of injection of the embolic agent is required. To prevent the occurrence of reflux, the balloon should be filled and complete obstruction of the target hepatic vein confirmed by angiography, prior to injection of the embolic agent. Immediate removal of the balloon catheter following occurrence of vascular casting in the target hepatic vein may prevent adhesion between the embolic agent and the balloon. The majority of studies use a ratio of 1:3-1:5 for the NBCA-lipiodol mixture $(15,16)$. However, according to the canine hepatic vein diameter and flow rate, a ratio of $2: 1$ was used in the present study for the NBCA-lipiodol mixture. A high percentage of NBCA results in rapid coagulation and adhesion with the balloon, while a low percentage causes slow coagulation, incomplete obstruction of the hepatic vein, reflux and pulmonary embolism. All animals in this study showed no adverse effects or serious complications of pulmonary embolism and mortality, indicating that a ratio of 2:1 for the NBCA-lipiodol mixture is suitable for establishment of a BCS model. The NBCA-lipiodol embolic agent has the advantage of rapid coagulation in the vessels, easy filling of the hepatic vein, tight connection with the hepatic vein wall and notable angiographic performance. Furthermore, the degree of hepatic vein obstruction may be easily controlled using an NBCA-lipiodol mixture as the embolic agent, which ensures that pulmonary embolism does not occur frequently. Therefore, obstruction of hepatic veins by a combination of balloon occlusion and NBCA-lipiodol as the embolic agent provides a novel, reliable and reproducible approach to developing a BCS animal model.

Canines possess 4-6 hepatic veins, which is different to the human liver anatomical structure. Dual-phase CT angiography of normal canine hepatic vasculature has demonstrated that several large hepatic vein branches, which drain the left lobes of the liver, anastomose to form a main trunk (17). In the present study, intraoperative angiography and postoperative anatomy confirmed that canine left and middle hepatic 
A

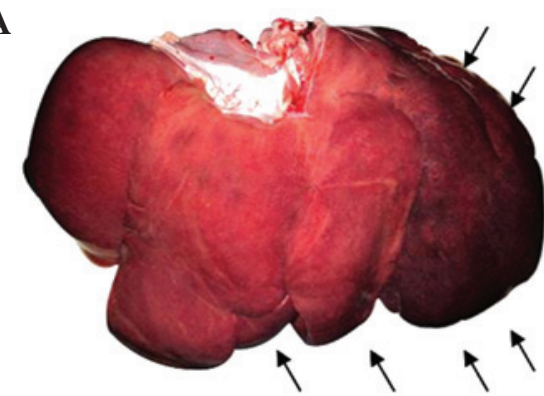

B

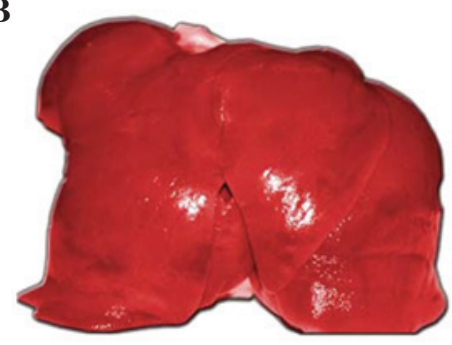

Figure 3. Morphological observations of canines. (A) Morphological examination of the liver showed hepatic lobe swelling with dark-red color in the target hepatic vein drainage area following 6 weeks of model development (arrows). (B) Morphological examination in the control group showed the liver to be bright red with a smooth surface and sharp edge.
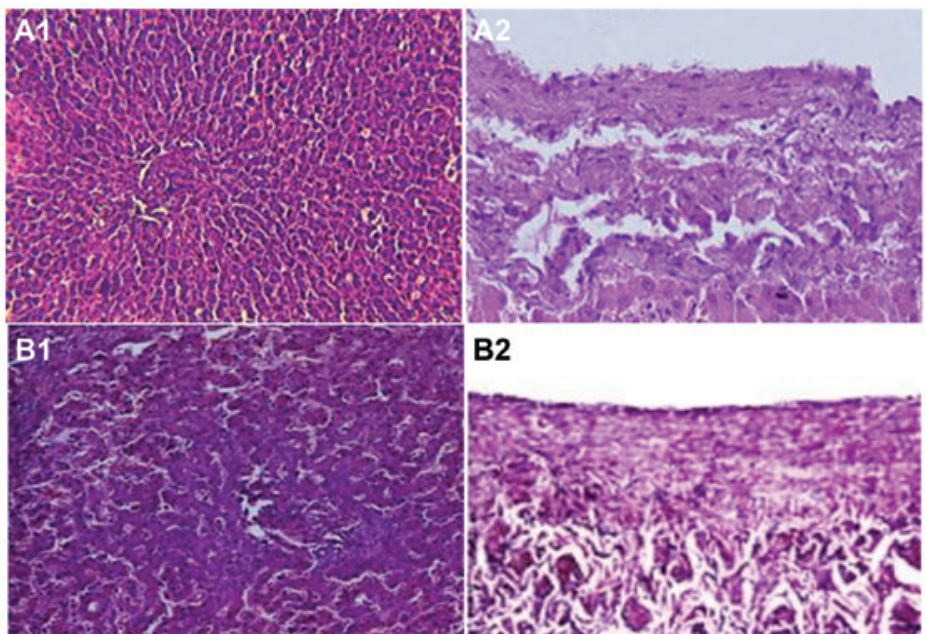

B2
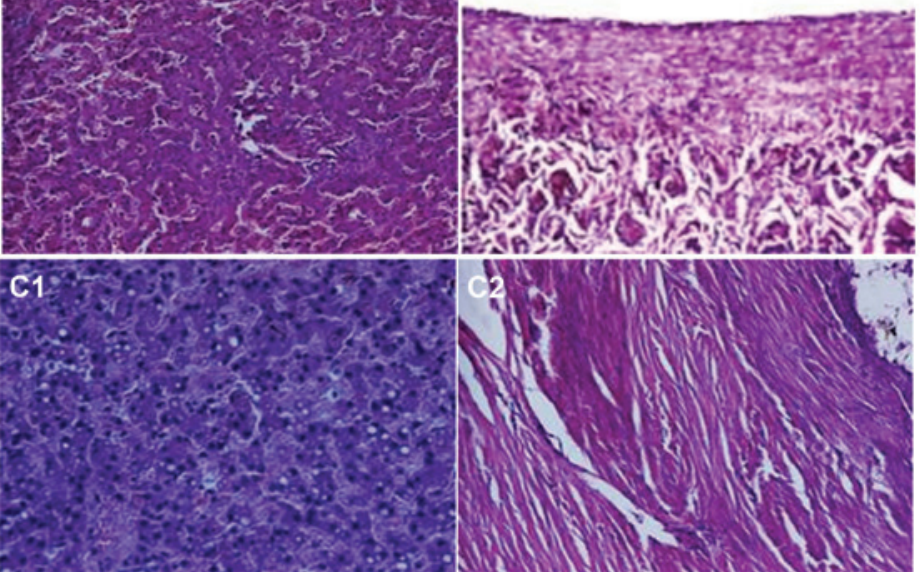

D1
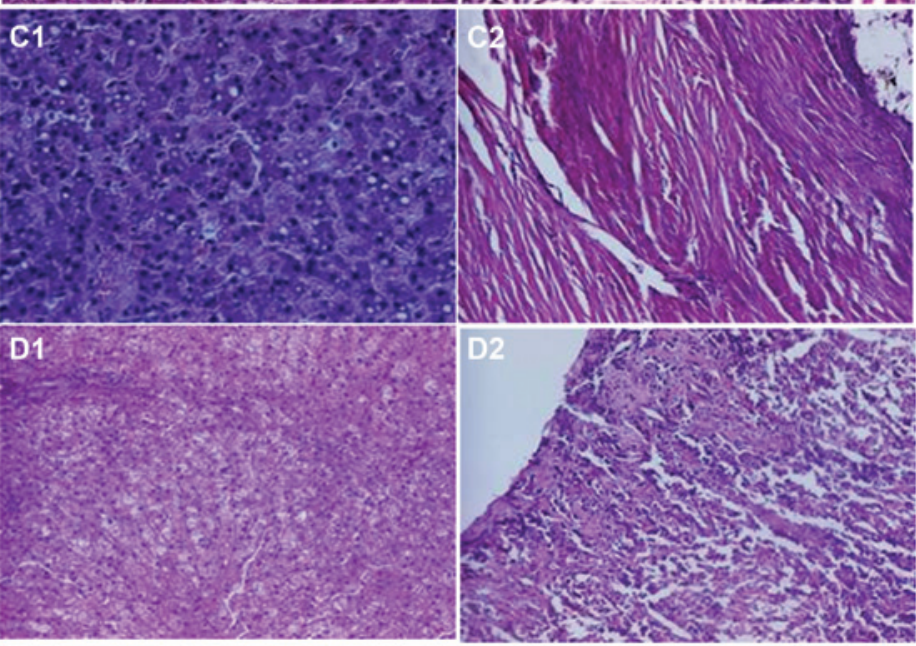

Figure 4. Histological observations of canine Budd-Chiari syndrome models and sham-treated canines (stain, hematoxylin and eosin; magnification, x200). $\left(A_{1}\right)$ Light microscopy shows normal hepatic cells in the control group following 4 weeks. $\left(A_{2}\right)$ Light microscopy shows the normal hepatic vein in the control group following 4 weeks. $\left(B_{1}\right)$ At week 4 of model development, light microscopy shows hepatic cell congestion, edema, dilated centrilobular sinusoids and a large quantity of inflammatory cell infiltration. $\left(\mathrm{B}_{2}\right)$ Inflammatory cell infiltration in the hepatic vein intima and tunica media without significant thickening at week 4 of model development. $\left(C_{1}\right)$ Liver histology shows hepatocyte swelling with lipid degeneration in target hepatic vein drainage area following 6 weeks of model development. $\left(\mathrm{C}_{2}\right)$ Histopathology of the target hepatic vein shows thickening of the tunica media smooth muscle and an increased number of layers following 6 weeks of model development. $\left(\mathrm{D}_{1}\right)$ Balloon-like changes of hepatocytes, increased size of nucleoli and increased number of cells with polyploid nucleus following 8 weeks of model development. $\left(\mathrm{D}_{2}\right)$ Significant hyperplasia and reduced inflammatory infiltration in collagen and elastic fibers of the target hepatic vein tunica media following 8 weeks of model development.

veins had a common trunk with the largest diameter among the hepatic veins and mainly collects venous blood from the left hepatic lobe, left central lobe, lobus quadratus and the majority of the right central lobe. By contrast, the right hepatic vein is small and only collects venous blood from the right dorsal part of the right hepatic lobe. Complete obstruction of all major hepatic veins may result in acute liver failure and mortality (18). However, the main characteristic of BCS is 
obstruction of the hepatic vein outflow tract. BCS is caused by occlusion of one, two or three of the major hepatic veins (right, middle and left) and/or occlusion of the inferior vena cava (19). The present study used the common trunk of left and middle hepatic veins as the site of occlusion and achieved severe obstruction of the outflow tract consistent with a diagnosis of BCS. Akiyoshi et al (11) used a surgical approach to ligate the inferior vena cava between the diaphragm muscle and liver in mice and observed severe hepatic congestion and mild fibrosis following one week of surgery. Following two weeks of surgery, this fibrosis had extended from the centrilobular zone to the midlobular zone (11). In the present study, significant damage to liver function was observed at weeks 4 and 6 following surgery, but had partially recovered by week 8 . Additionally, liver swelling and congestion, hepatocyte degeneration and necrosis tended to be worse between 4-8 weeks following surgery. Typical histopathology observed with obstruction of the human hepatic venous outflow tract includes hepatic congestion and fibrosis and loss of liver cells (20). Histopathology of the canine BCS model in this study was similar to that of the mouse BCS model and to human BCS. Previous studies have shown that necrotic hepatocytes are gradually replaced by fibrosis, eventually resulting in cirrhosis $(18,21)$. The development of cirrhosis from hepatic fibrosis takes a relatively long time. For instance, it takes 2-15 years (average, 8.7 years) for congestive liver fibrosis of the hepatic vena cava disease (a form of BCS) to advance to cirrhosis (22). It was hypothesized that, with time, cirrhosis may also develop following hepatocyte necrosis in the canine BCS model of the present study.

Hepatic vein obstruction leads to the elevation of sinusoidal blood pressure and portal vein pressure and ultimately portal vein hypertension. Hiraki et al (23) showed that maximum peak velocity of the portal vein was reduced and Doppler signals disappeared following temporary occlusion of the right hepatic vein in humans, indicating that portal vein hemodynamics change shortly following hepatic vein obstruction. In the present study, average peak velocity of the main portal vein and portal blood flow volume decreased significantly at 4-8 weeks following surgery, which indicated an increased resistance of the portal vein. With time, BCS patients may form intrahepatic venous and portosystemic collateral circulations, which partially alleviate elevated portal vein pressure (24-26). Compensatory mechanisms of portal veins were also observed in the mouse BCS model (10). However, in the present study, the portal vein pressure was altered significantly following 4,6 or 8 weeks of hepatic vein obstruction. Upper gastrointestinal bleeding caused by esophageal varices was observed in one canine BCS model at 6 weeks following surgery. Results of the present study demonstrate that embolization of the largest hepatic vein in canines has a significant effect on portal vein hemodynamics.

In a previous study, average peak velocity of the right hepatic artery was found to decrease marginally for 15-30 sec following the onset of hepatic vein occlusion, prior to increasing rapidly by 1.5-2.0 times at 75-90 sec. Once the hepatic vein became unobstructed, average peak velocity began to decrease (23). However, in the present study, hepatic vein occlusion was permanent and the RI of the proper hepatic artery increased following 4-8 weeks of hepatic vein obstruction. The increase in RI following surgery may be explained by an elevation in peripheral vascular resistance, which is caused by an increase in sinusoidal pressure due to liver congestion and hepatic cell necrosis.

One limitation of this study was that there was only obstruction of the hepatic vein with the largest diameter (the common trunk of left and middle hepatic veins), while the other hepatic veins remained unobstructed. Future studies must investigate the obstruction of the remaining hepatic veins following a given time period for obstruction of the largest hepatic vein. This is likely to allow recovery of liver function and formation of a compensatory collateral circulation in order to avoid fulminant hepatic failure and mortality observed in human acute hepatic vein obstruction $(27,28)$. In addition, the long-term histopathological and hemodynamic changes following the development of a BCS model also require further study.

In conclusion, the present study successfully established a canine BCS model using a balloon and NBCA-lipiodol mixture to obstruct hepatic veins. This model is reliable, reproducible and produces pathophysiological changes consistent with human hepatic vein obstruction. In addition, compared with the BCS model developed by surgical procedures, endovascular obstruction is advantageous due to minimal trauma, fewer incidences of complications and a greater ease of implementation. Due to diffuse hepatic vein occlusion, this canine BCS model may also be useful to studies regarding angiogenesis (e.g., endothelial progenitor cells, vascular endothelial growth factor), surgery and interventional radiology.

\section{Acknowledgements}

This study was financially supported by grants from Jiangsu Province (333 Project; no. BRA2011221), Xuzhou Municipal Science and Technology Bureau (grant no. XF11C097) and Xuzhou Medical College Dean Special Talent Fund (grant no. 2011KJZ27).

\section{References}

1. Janssen HL, Garcia-Pagan JC, Elias E, Mentha G, Hadengue A and Valla DC; European Group for the Study of Vascular Disorders of the Liver: Budd-Chiari syndrome: a review by an expert panel. J Hepatol 38: 364-371, 2003.

2. Valla DC: Primary Budd-Chiari syndrome. J Hepatol 50: 195-203, 2009.

3. Horton JD, San Miguel FL, Membreno F, et al: Budd-Chiari syndrome: illustrated review of current management. Liver Int 28: 455-466, 2008.

4. Cura M, Haskal Z and Lopera J: Diagnostic and interventional radiology for Budd-Chiari syndrome. Radio Graphics 29: 669-681, 2009.

5. Plessier A and Valla DC: Budd-Chiari syndrome. Semin Liver Dis 28: 259-269, 2008.

6. Zeitoun G, Escolano S, Hadengue A, et al: Outcome of Budd Chiari syndrome: a multivariate analysis of factors related to survival including surgical portosystemic shunting. Hepatology 30: 84-89, 1999.

7. Darwish Murad S, Valla DC, de Groen PC, et al: Determinants of survival and the effect of portosystemic shunting in patients with Budd-Chiari syndrome. Hepatology 39: 500-508, 2004.

8. Langlet P, Escolano S, Valla D, et al: Clinicopathological forms and prognostic index in Budd-Chiari syndrome. J Hepatol 39: 496-501, 2003.

9. Garcia-Pagán JC, Heydtmann M, Raffa S, et al: TIPS for Budd Chiari syndrome: long-term results and prognostics factors in 124 patients. Gastroenterology 135: 808-815, 2008. 
10. Darwish Murad S, Dom VA, Ritman EL, et al: Early changes of the portal tract on microcomputed tomography images in a newly-developed rat model for Budd-Chiari syndrome. J Gastroenterol Hepatol 23: 1561-1566, 2008.

11. Akiyoshi $\mathrm{H}$ and Terada T: Centrilobular and perisinusoidal fibrosis in experimental congestive liver in the rat. J Hepatol 30 433-439, 1999.

12. Pancholy SB, Sanghvi KA and Patel TM: Radial artery access technique evaluation trial: randomized comparison of Seldinger versus modified Seldinger technique for arterial access for transradial catheterization. Catheter Cardiovasc Interv 80: 288-291, 2012.

13. de Baere T, Deschamps F, Briggs P, et al: Hepatic malignancies: percutaneous radiofrequency ablation during percutaneous portal or hepatic vein occlusion. Radiology 248: 1056-1066, 2008.

14. Wang MQ, Duan F, Liu FY, Wang ZJ and Song P: Treatment of symptomatic polycystic liver disease: transcatheter super-selective hepatic arterial embolization using a mixture of NBCA and iodized oil. Abdom Imaging 38: 465-473, 2013.

15. Yoo DH, Jae HJ, Kim HC, Chung JW and Park JH: Transcatheter arterial embolization of intramuscular active hemorrhage with N-butyl cyanoacrylate. Cardiovasc Intervent Radiol 35: 292-298, 2012.

16. Loewe C, Schindl M, Cejna M, Niederle B, Lammer J and Thurnher S: Permanent transarterial embolization of neuroendocrine metastases of the liver using cyanoacrylate and lipiodol: assessment of mid- and long-term results. Am J Roentgenol 180 $1379-1384,2003$.

17. Zwingenberger AL and Schwarz T: Dual-phase CT angiography of the normal canine portal and hepatic vasculature. Vet Radiol Ultrasound 45: 117-124, 2004

18. Hoekstra J and Janssen HL: Vascular liver disorders (I): diagnosis, treatment and prognosis of Budd-Chiari syndrome. Neth J Med 66: 334-339, 2008

19. Mine T: Is hepatic vena cava disease an endemic type of the Budd-Chiari syndrome? Hepatol Res 37: 170-171, 2007.
20. Tanaka M and Wanless IR: Pathology of the liver in Budd-Chiari syndrome: portal vein thrombosis and the histogenesis of veno-centric cirrhosis, veno-portal cirrhosis, and large regenerative nodules. Hepatology 27: 488-496, 1998

21. Cazals-Hatem D, Vilgrain V, Genin P, et al: Arterial and portal circulation and parenchymal changes in Budd-Chiari syndrome: a study in 17 explanted livers. Hepatology 37: 510-519, 2003.

22. Shrestha SM: Liver cirrhosis and hepatocellular carcinoma in hepatic vena cava disease, a liver disease caused by obstruction of inferior vena cava. Hepatol Int 3: 392-402, 2009.

23. Hiraki T, Kanazawa S, Mimura H, et al: Altered hepatic hemodynamics caused by temporary occlusion of the right hepatic vein: evaluation with Doppler US in 14 patients. Radiology 220: 357-364, 2001.

24. Küşük NO, Ozkan E, Aras G and Kir KM: Visualization of collaterals in budd-chiari syndrome with Tc-99m MDP bone scintigraphy and Tc-99m HMPAO-labeled leukocyte scintigraphy. Clin Nucl Med 28: 236-237, 2003.

25. Steingruber IE, Bodner G, Czermak B, Hochleitner B and Jaschke W: Color-coded doppler sonographic demonstration of intrahepatic venous collaterals in Budd-Chiari syndrome. Ultraschall Med 22: 55-59, 2001 (In German).

26. Karaosmanoglu D, Karcaaltincaba M, Akata D, Ozmen M and Akhan O: CT, MRI, and US findings of incidental segmental distal hepatic vein occlusion: a new form of Budd-Chiari syndrome? J Comput Assist Tomogr 32: 518-522, 2008.

27. Shih KL, Yen HH, Su WW, Soon MS, Hsia CH and Lin YM: Fulminant Budd-Chiari syndrome caused by renal cell carcinoma with hepatic vein invasion: report of a case. Eur J Gastroenterol Hepatol 21: 222-224, 2009.

28. Amitrano L, Guardascione MA, Schiavone EM, et al: Hepatic vein thrombosis leading to fulminant hepatic failure in a case of acute non-promyelocytic myelogenous leukemia. Blood Coagul Fibrinolysis 17: 59-61, 2006. 